\title{
Análise do sistema nervoso autônomo de indivíduos jovens com diabetes mellitus tipo 1
}

\author{
Análisis del sistema nervioso de los individuos jóvenes con diabetes mellitus tipo 1 \\ Analysis of the autonomic nervous system of young individuals with type 1 diabetes \\ mellitus
}

Ana Paula Ferreira ${ }^{1}$, Plínio dos Santos Ramos ${ }^{1}$, Djalma Rabelo Ricardo ${ }^{1}$, Heitor Motta Bini Pereira ${ }^{1 *}$, Matheus Monteiro de Oliveira ${ }^{2}$, Lucas Monteiro de Oliveira², Túlio Gonçalves dos Reis³.

\section{RESUMO}

Objetivo: Analisar o comportamento do sistema nervoso autônomo (SNA) de indivíduos com Diabetes Mellitus tipo 1 (DM1). Métodos: Trata-se de um estudo descritivo transversal, que investigou 35 indivíduos do sexo masculino, com idade entre 18 a 30 anos, divididos em dois grupos a saber: GDM1 - 19 jovens com diabetes mellitus tipo 1, com tempo de exposição à doença de $13 \pm 6,89$ anos (média \pm desvio padrão) e GC - 16 indivíduos saudáveis. Os dois grupos foram submetidos aos testes de variabilidade da frequência cardíaca (VFC) e teste de exercício de quatro segundos (T4s) que avalia isoladamente o ramo parassimpático do SNA, sendo seu resultado expresso pelo índice vagal cardíaco (IVC). Resultados: Os índices do domínio do tempo e da frequência por meio da VFC não apresentaram diferenças ( $p>0.05)$, assim como IVC ( $p=0,33)$. Todavia a Frequência Cardíaca (FC) de repouso registrada durante a VFC, apresentou diferenças significativas entre o GDM1 e o GC ( $p=0,0001)$. Conclusão: Os achados do presente estudo, contribuem por reforçar que identificar de forma precoce a disfunção autonômica em indivíduos com DM, pode acelerar a profilaxia e o controle de lesões em órgãos alvos com o uso de drogas específicas e também com abordagens não farmacológicas.

Palavras-chave: Diabetes mellitus tipo 1, Sistema nervoso autônomo, Frequência cardíaca.

\begin{abstract}
Objective: To analyze the behavior of the autonomic nervous system (ANS) of individuals with type 1 Diabetes Mellitus (DM1). Methods: This is a descriptive cross-sectional study, which investigated 35 male individuals, aged between 18 and 30 years old, divided into two groups, namely: GDM1 - 19 young people with type 1 diabetes mellitus, with time of exposure to the disease aged $13 \pm 6.89$ years (mean \pm standard deviation) and CG - 16 healthy individuals. Both groups underwent heart rate variability (HRV) tests and a four-second exercise test (T4s) that separately assesses the parasympathetic branch of the ANS, the result of which is expressed by the cardiac vagal index (CVI). Results: The time and frequency domain indexes using HRV did not differ $(p>0.05)$, as well as CVI $(p=0.33)$. However, the resting heart rate $(H R)$ recorded during HRV, showed significant differences between GDM1 and CG $(p=0.0001)$. Conclusion: The findings of the present study contribute to reinforce that early identification of autonomic dysfunction in individuals with DM can accelerate the prophylaxis and control of lesions in target organs with the use of specific drugs and also with non-pharmacological approaches.
\end{abstract}

Keywords: Diabetes mellitus type 1, Autonomic nervous system, Heart rate.

\footnotetext{
${ }^{1}$ Faculdade de Ciências Médicas e da Saúde de Juiz de Fora, Juiz de Fora - MG.

*E-mail: heitorbini3@gmail.com

2 Universidade Federal de Juiz de Fora, Juiz de Fora - MG.

${ }^{3}$ Santa Casa de Misericórdia de Juiz de Fora, Juiz de Fora - MG.
} 


\section{RESUMEN}

Objetivo: Analizar el comportamiento del sistema nervioso autónomo (SNA) de las personas con diabetes mellitus tipo 1 (DM1). Métodos: Este es un estudio descriptivo de corte transversal, que investigó a 35 individuos varones, con edades comprendidas entre 18 y 30 años, divididos en dos grupos, a saber: GDM1 19 jóvenes con diabetes mellitus tipo 1, con tiempo de exposición a la enfermedad. edad $13 \pm 6.89$ años (media \pm desviación estándar) y CG - 16 individuos sanos. Ambos grupos se sometieron a pruebas de variabilidad de la frecuencia cardíaca (VFC) y una prueba de ejercicio de cuatro segundos (T4) que evalúa por separado la rama parasimpática del SNA, cuyo resultado se expresa mediante el índice vagal cardíaco (IVC). Resultados: Los índices de dominio de tiempo y frecuencia usando HRV no fueron diferentes ( $p>0.05$ ), así como CVI $(p=0.33)$. Sin embargo, la frecuencia cardíaca en reposo $(F C)$ registrada durante la VFC mostró diferencias significativas entre GDM1 y CG $(p=0,0001)$. Conclusión: Los hallazgos del presente estudio contribuyen a reforzar que la identificación temprana de la disfunción autonómica en individuos con DM puede acelerar la profilaxis y el control de las lesiones en órganos diana con el uso de medicamentos específicos y también con enfoques no farmacológicos.

Palabras clave: Diabetes mellitus tipo 1, Sistema nervioso autónomo, Frecuencia cardíaca.

\section{INTRODUÇÃO}

O diabetes mellitus tipo 1 (DM1), é uma doença crônica de caráter autoimune, caracterizada pela produção insuficiente de insulina pelo pâncreas, sendo responsável por inúmeras complicações em diversos órgãos e sistemas do indivíduo (SCHIMID H, 2007). Dentre estas complicações, destacamos as que acometem o sistema nervoso autônomo (SNA), como por exemplo as neuropatias autonômicas diabéticas, que podem levar a um aumento importante das taxas de morbidade e mortalidade nesta população (DE ANGELIS K, et al., 2009).

De acordo com Maser RE, et al (2003), os distúrbios metabólicos são os principais preditores das alterações da função autonômicas, conduzindo o aparecimento de sinais e sintomas como intolerância ao exercício, taquicardia de repouso, prolongamento do intervalo QT, variação anormal da pressão arterial, isquemia miocárdica silenciosa e cardiomiopatia diabética (ROLIM LC, et al., 2008 e POP-BUSUI R, et al., 2010).

Os acometimentos neurológicos são extensos no indivíduo com DM1, podendo alterar os componentes sensoriais, motores e autonômicos do sistema nervoso, com patogênese pouco clara, mas fortemente relacionada a alterações de origem metabólica e/ou doença microvascular (ABD EL DAYEM SM, et al., 2011).

No estágio inicial da doença, o nervo vago, tende a ser o primeiro acometido, levando a uma redução da atividade parassimpática que em contrapartida acaba resultando em um aumento da atividade simpática. Esta disfunção autonômica por sua vez, pode ser identificada logo nos estágios iniciais da doença independente de fatores prévios de risco cardiovascular (JAYORKA M, et al., 2008).

Apesar das disfunções autonômicas estarem entre as complicações crônicas mais graves e de pior prognóstico do DM1, pouco se sabe sobre sua progressão natural (KARAMITSOS DT, et al., 1998). Estudos longitudinais que investigaram o acometimento do SNA nesta população, verificaram piora importante do controle autonômico em função de controle glicêmico ruim e tempo de exposição à doença (ZANONE MM, et al., 2003).

Contudo, em indivíduos com DM1 tais alterações devem ser rastreadas precocemente, mesmo na ausência de sintomas, a partir de cinco anos de diagnóstico (SPALLONE V, et al., 2011). Um aspecto relevante a se destacar, é que a maioria dos estudos que se debruçaram sobre tal temática investigaram a modulação autonômica em especial pela variabilidade da frequência cardíaca (VFC) e pela frequência cardíaca (FC) de repouso.

Todavia, poucos estudos se dedicaram a investigar isoladamente alterações no ramo parassimpático do SNA, ou seja, a atividade vagal cardíaca (AVC) permanecendo ainda desta forma um óbice perante a comunidade científica. Dessa forma, o objetivo do presente estudo foi analisar o comportamento do sistema nervoso autônomo (SNA) de indivíduos com DM1. 


\section{MÉTODOS}

\section{Amostra}

Trata-se de um estudo de natureza descritiva, do tipo transversal, no qual participaram 35 indivíduos do sexo masculino com idade entre 18 e 30 anos, sendo estes divididos em dois grupos: grupo DM1 (GDM1) com 19 indivíduos com DM1 e grupo controle (GC) com 16 indivíduos saudáveis com média de idade $(23,11$ $\pm 3,28$ versus $23,83 \pm 3,63$, respectivamente, $p=0,17$; média \pm desvio padrão). Foram incluídos no GDM1 os indivíduos que não apresentavam diagnóstico de neuropatia prévia e, com tempo de exposição à doença superior a cinco anos.

Foram excluídos, os sujeitos com complicações diabéticas agudas como cetoacidose diabética ou hipoglicemia, com diagnóstico de doenças cardíacas congênitas ou reumáticas, disfunção ventricular esquerda ou hipertensão, insuficiência renal crônica e distúrbios eletrolíticos, incapacidade de realizar os protocolos, obesidade (IMC $\left.>30 \mathrm{~kg} / \mathrm{m}^{2}\right)$, e que estavam fazendo uso de medicações passíveis de interferência no SNA.

Para a constituição do GC a inclusão dos voluntários foi realizada, após um teste para avaliação dos níveis glicêmicos em jejum com o glicosímetro (Abbott, FreeStyleOptium H, WitneyOxforshire, Reino Unido), realizado no período da manhã, e foram incluídos apenas os que apresentavam os níveis de glicose em jejum $<100 \mathrm{mg} / \mathrm{dL}^{2}$. Todos os voluntários do estudo leram e assinaram um termo de consentimento livre e esclarecido, previamente aprovado pelo comitê de ética em pesquisa institucional (parecer úmero 148/10), de acordo com a resolução 466/12 do Conselho Nacional de Saúde.

\section{Procedimento de coleta de dados}

Os indivíduos da pesquisa realizaram uma visita ao laboratório de fisiologia para realização da avaliação da FC de repouso, da AVC e da VFC. Os indivíduos foram orientados a não ingerir bebidas cafeinadas e alcoólicas, bem como não realizar exercício físico intenso nas últimas 24 horas e não ingerir alimentos e líquidos nas duas horas anteriores a realização dos testes.

Inicialmente foi realizada uma anamnese, que incluía informações sobre o histórico médico, uso de medicamentos, tabagismo, prática de exercícios físicos e dose diária de insulina. Foram definidos tabagistas aqueles que fumavam $\geq 1$ cigarros nos 30 dias anteriores à pesquisa, e em relação a prática de exercícios físicos, foram considerados fisicamente ativos aqueles que realizavam de 30 a 60 minutos de exercício físico por dia, ou 150 minutos por semana (GARBER CE, et al., 2011).

A medida da altura foi realizada por meio de um estadiômetro com precisão milimétrica (Sanny, Brasil) e o peso corporal foi avaliado usando uma balança de peso Digital (Welmy, Brasil), com precisão de 0,1 kg. O IMC foi calculado como peso em quilogramas dividido pelo quadrado da altura em metros $\left(\mathrm{Kg} / \mathrm{m}^{2}\right)$. Em seguida, foi medida a pressão arterial em repouso de acordo com o protocolo da $V$ Diretriz Brasileira de Hipertensão (MACHADO CA, et al., 2007).

A avaliação da modulação autonômica cardíaca foi realizada por meio da FC de repouso, análise da VFC e pelo teste de exercício de quatro segundos (T4s), que foi realizado em cicloergômetro de membros inferiores, sem carga, durante uma apneia inspiratória máxima de 12 segundos.

\section{Frequência Cardíaca (FC) de repouso}

A média da FC de repouso foi obtida por meio de um traçado eletrocardiográfico feito nas derivações (CC5 ou CM5) utilizando o sistema PowerLab (PowerLab 4 / 25T e Laboratório Gráfico 7 software Pro; ADInstruments, Austrália).

\section{Variabilidade da frequência cardíaca}

Os participantes foram avaliados seguindo os critérios preconizados pela Sociedade Européia de Cardiologia e a Sociedade Norte-Americana de Marca-Passos e Eletrofisiologia. A análise de VFC, foi realizada em repouso, na posição supina, por um período de 20 minutos, sendo os 10 minutos iniciais utilizados para estabilização dos intervalos RR e os 10 minutos seguintes para registro e análise. 
A partir do registro do eletrocardiograma (PowerLab 4/25T, ADInstruments, Austrália) foram identificados os intervalos entre ondas $R$ de batimentos sinusais com precisão de $1 \mathrm{~ms}$. Foram realizadas análises do domínio do tempo e frequência (VANDERLEI LC, et al., 2009).

No domínio do tempo a modulação vagal cardíaca foi avaliada pelos índices SDNN, desvio padrão de todos os intervalos RR normais gravados em um intervalo de tempo, pelo rMSSD, raiz quadrada da média do quadrado das diferenças entre os intervalos $R R$ normais adjacentes e pNN50, que representa a porcentagem dos intervalos RR adjacentes com diferenças de duração maior que $50 \mathrm{~ms}$. Já no domínio da frequência, a densidade de potência espectral da banda de alta e baxa frequência foram realizadas em unidades normalizadas (AF u.n.) (VANDERLEI LC, et al., 2009).

\section{Teste de exercício de 4 segundos}

O T4s possui o objetivo de avaliar separadamente a integridade do ramo parassimpático. $\mathrm{O}$ teste se formaliza quando o paciente ao pedalar de forma acelerada em um ciclo ergômetro sem carga, do 5ำ ao 9o segundo de uma apnéia inspiratória máxima com duração de 12 segundos.

É preciso apontar que o T4s quantifica a AVC por meio do IVC e essa significa a aceleração da frequência cardíaca desencadeada reflexamente pela inibição vagal cardíaca, onde através dos intervalos RR do eletrocardiograma são analisados os intervalos RR obtidos pela duração dos ciclos cardíacos. Ademais, é necessário enfatizar que durante o teste, o paciente segue quatro comandos consecutivos dados pelo avaliador: no $1^{\circ}$ ele realiza uma inspiração máxima e rápida pela boca; no $2^{\circ}$ pedala de forma acelerada; no 3 ำ para de pedalar bruscamente e no $4^{\circ}$ comando, ele realiza a expiração (ARAÚJO CGSD, et al., 2003).

Cabe ressaltar que o IVC, índice adimensional obtido pelo T4s que é um dado de extrema confiança, com alta especificidade para análise e de extrema importância para a validade da atual pesquisa. Além disso, o IVC possui teor farmacológico, ou seja, também é validado de farmacologicamente e é obtido pela razão entre o intervalo RR imediatamente antes ou o primeiro do exercício, geralmente o mais longo (RRB), e o intervalo RR mais curto durante o exercício, geralmente o último (RRC) (Figura 1). Para o registro do eletrocardiograma durante o T4s foi utilizado o mesmo aquisitor de sinal biológico das variáveis anteriormente coletadas.

Figura 1 - Identificação e medida dos intervalos RRB, RRC e cálculo do IVC.

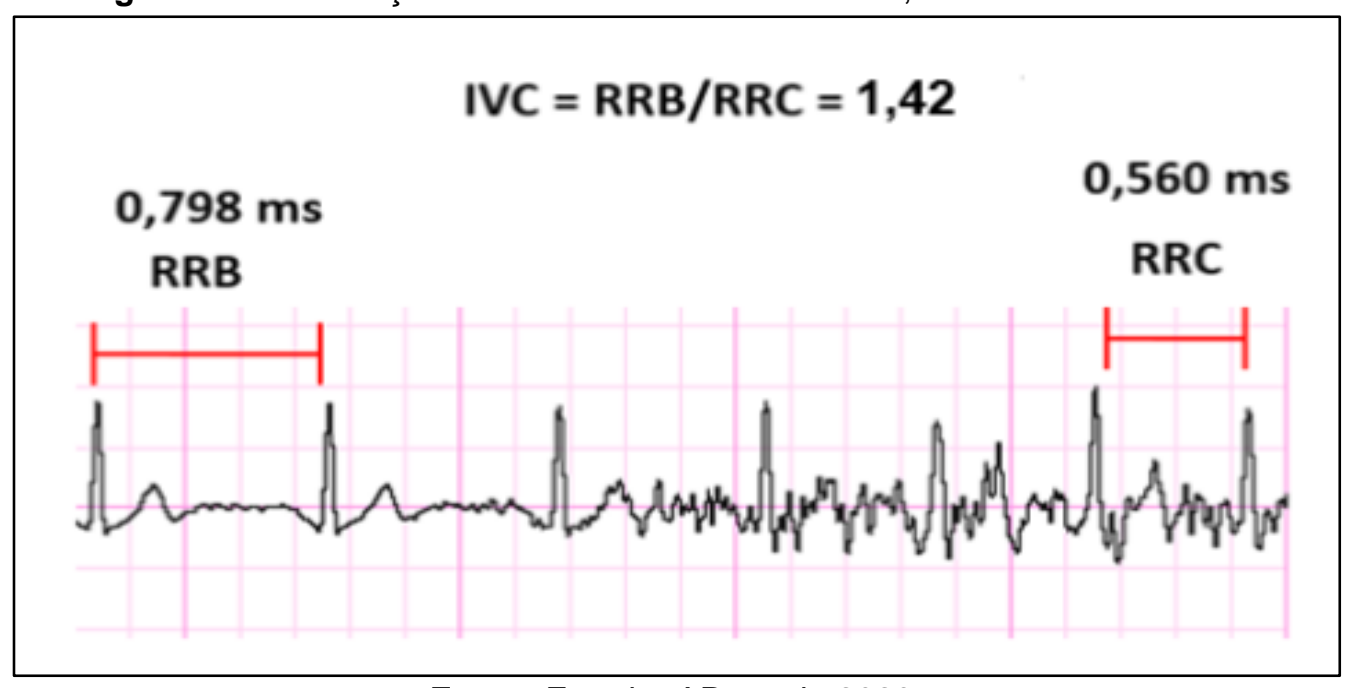

Fonte: Ferreira AP, et al., 2020.

\section{Análise estatística}

Inicialmente foi testada a normalidade dos dados pelo teste de Shapiro Wilk. Para as variáveis consideradas de distribuição normal, foi utilizado o teste-t de Student para comparar os resultados encontrados no grupo controle e no grupo de pacientes com DM1. Para as variáveis cuja distribuição não caracterizou uma distribuição normal no teste de Shapiro Wilk foi realizado o teste de Mann Whitney, a fim de comparar os resultados entre os grupos estudados. 
Foi aceito como nível de significância $p>0,05$ e intervalo de confiança de $95 \%$. Foi utilizado o software estatístico GraphPad (versão 5.01, GraphPad, La Jolla CA) e RStudios (versão 1.2.5001, RStudios) para o tratamento dos dados. As análises descritivas dos dados foram apresentadas por média e desvio padrão da média.

\section{RESULTADOS}

Os 19 indivíduos com DM1 apresentavam como tempo médio de exposição à doença $13 \pm 6,8$ anos (média \pm desvio padrão). Os dados referentes as variáveis antropométricas e hemodinâmicas dos indivíduos avaliados são apresentados (Tabela 1). Não houve diferenças estatisticamente significativas entre os indivíduos do GDM1 e do GC*.

Tabela 1 - Variáveis antropométricas e hemodinâmicas dos grupos estudados

\begin{tabular}{cccc}
\hline \multirow{2}{*}{ Variáveis } & $(\mathbf{G C}=\mathbf{1 6})$ & \multirow{2}{*}{ Valor de $\mathbf{p}$} \\
\cline { 2 - 3 } & Média $\pm \mathbf{D P}$ & Média $\pm \mathbf{D P}$ & 0,92 \\
\hline Peso $(\mathbf{k g})$ & $74,7 \pm 10,9$ & $67,1 \pm 8,5$ & 0,83 \\
Altura $(\mathbf{m})$ & $1,75 \pm 0,7$ & $1,73 \pm 0,7$ & 0,92 \\
IMC $\left(\mathbf{k g} / \mathbf{m}^{2}\right)$ & $24,2 \pm 2,1$ & $22,3 \pm 2,2$ & 0,51 \\
PAS $(\mathbf{m m H g})$ & $116 \pm 9,5$ & $115 \pm 12,2$ & 0,89 \\
PAD $(\mathbf{m m H g})$ & $75 \pm 10,9$ & $73 \pm 12,4$ &
\end{tabular}

Legenda: DP (desvio padrão); IMC (índice de massa corpórea); PAS (pressão arterial sistólica); PAD (pressão arterial diastólica).

Fonte: Ferreira AP, et al., 2020.

A proporção de indivíduos considerados fisicamente ativos nos dois grupos foi semelhante tanto no primeiro (GC - 50\% versus GDM1 - 42\%; $p=0,64$ ) quanto no segundo exame (GC - 68\% versus GDM1 - 47\%; $p=0,30$ ). A FC de repouso foi significativamente maior entre os jovens no grupo GDM1 quando comparado ao $G C$ dois exames realizados ( $p=0,031$ ) (Figura 2).

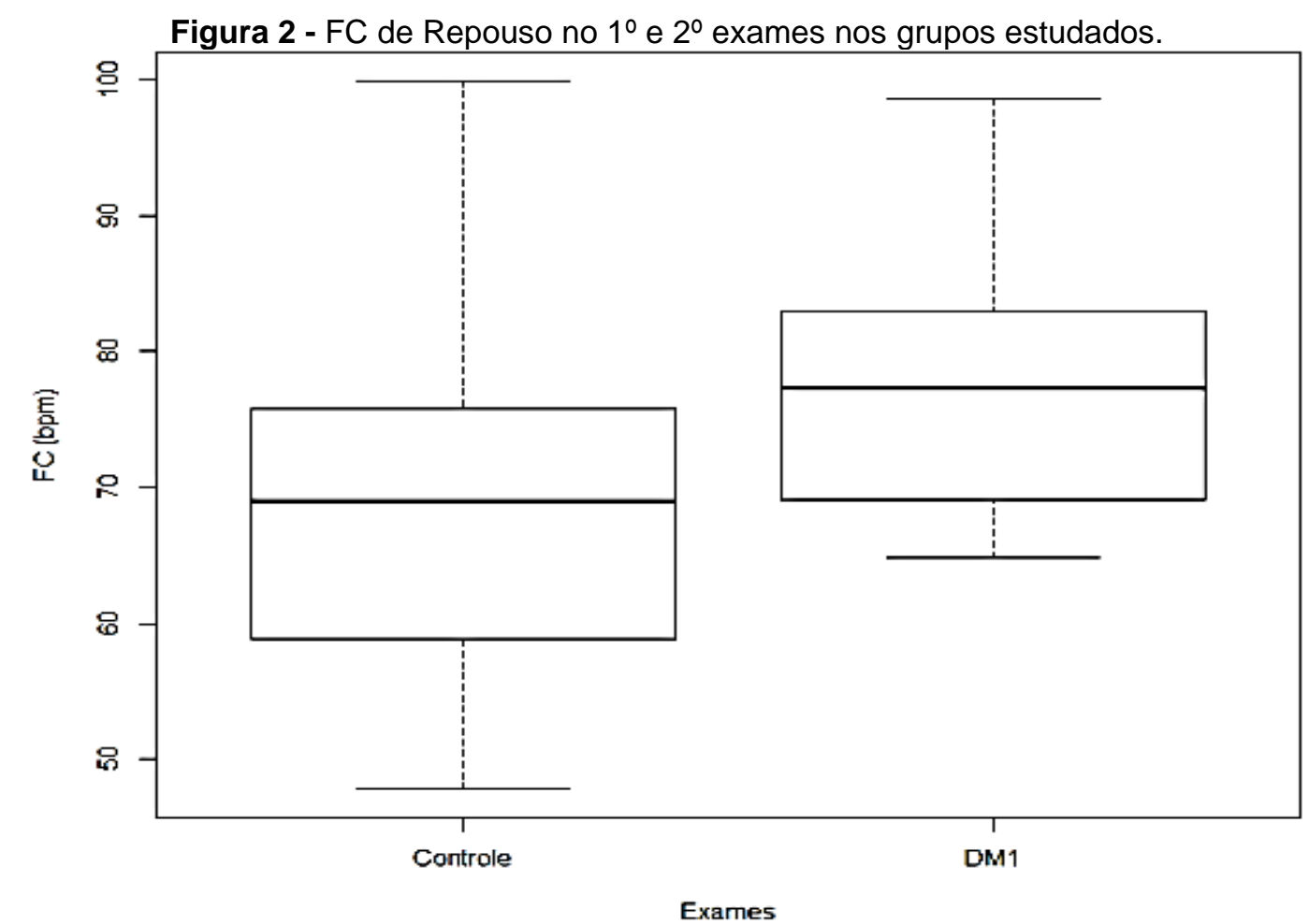

Legenda: ${ }^{*} \mathrm{p}=0,0001$ entre os grupos GCeGDM1.

Fonte: Ferreira AP, et al., 2020. 
Nos índices da VFC no domínio do tempo e da frequência não houve diferenças entre os grupos e entre as avaliações realizadas, conforme podemos observar na Tabela 2.

Tabela 2 - Variabilidade da Frequência Cardíaca (VFC) de índices obtidos por meio de métodos lineares no domínio do tempo e frequência em repouso.

\begin{tabular}{cccc}
\hline Variáveis & GC ( $\mathbf{n = 1 6 )}$ & GDM1 (n=19) & P valor \\
\hline Domínio de tempo & & & 0.502 \\
\hline SDNN (ms) & 96.34 & 118.43 & 0.481 \\
RMSSD (ms) & 100.25 & 110.42 & 0.276 \\
PMN50 (\%) & 209.60 & 153.10 & \\
\hline Domínio de frequência & & & 0.920 \\
\hline \%LF (n.u.) & 29.88 & 29.20 & 0.071 \\
\%HF (n.u.) & 63.54 & 50.04 & 0.301 \\
LF / HF & 0.68 & 0.70 & \\
\hline
\end{tabular}

Fonte: Ferreira AP, et al., 2020.

Dados apresentados como média * *diferenças em relação ao GC considerando nível de significância * p>0,05.O IVC obtido no T4s não apresentou diferenças entre os grupos estudados *(GDM1 - 1,38 \pm 0,19; GC - 1,42 $\pm 0,22, p=0.4482 ;$ média \pm desvio padrão da média). Contudo, o GDM1 apresentou um IVC médio 3\% menor que o GC (Figura 3).

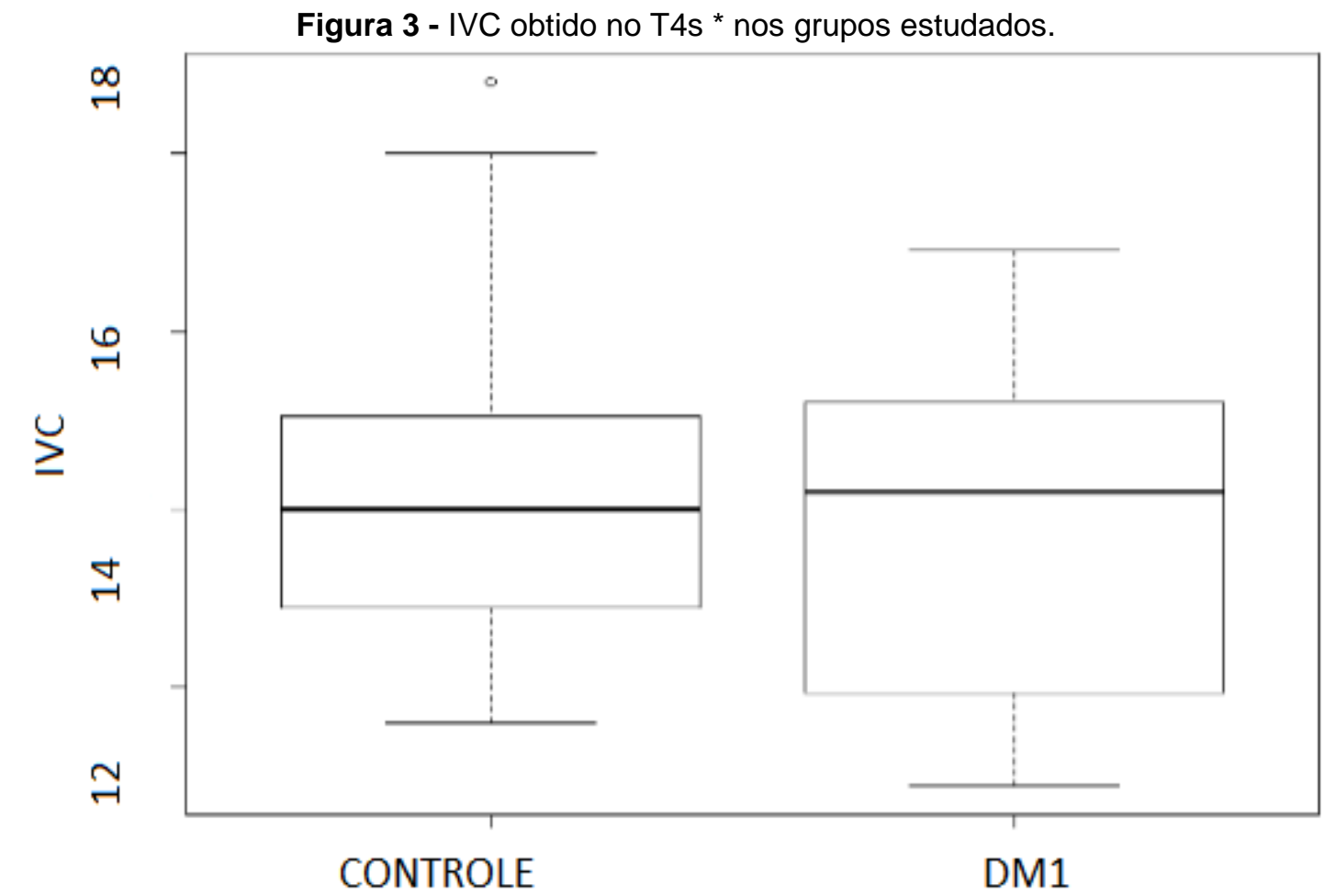

Fonte: Ferreira AP, et al., 2020. 


\section{DISCUSSÃO}

O presente estudo contribui com o corpo de conhecimento da área ao avaliar o SNA de indivíduos com DM através da FC de repouso, a VFC e a AVC, mostrando que o GDM1 apresentou uma menor FC de repouso, quando comparado ao controle. Por outro lado, as variáveis derivadas da VFC não apresentaram diferenças importantes em comparação ao GC. Apesar de não existir diferença estatisticamente significativa na avaliação isolada do ramo parassimpático do SNA, podemos observar um menor valor no IVC, cerca de 3\% no GDM1.

A chance de melhorar o prognóstico de um paciente diabético com alterações autonômicas é realizando o diagnóstico precoce. O tratamento da neuropatia autonômica cardiovascular (NAC) é baseado em abordagens não farmacológicos que incluem redução de peso e melhorias na resistência à insulina, uma vez que ambos estão associados à hiperatividade simpática (HOWORKA K, et al., 1997).

Medidas farmacológicas, como inibidores da enzima conversora de angiotensina (ECA) e betabloqueadores, podem contribuir para a atenuação dos sintomas da NAC, contudo terapias cujo foco é, exclusivamente, a ação do sistema nervoso simpático ainda requerem mais investigação, como (i) hiperestimulação do nervo vago; (ii) ablação simpática renal; (iii) ressecção do corpo carotídeo; e (iv) estimulação elétrica do barorreceptor (BREDER IS, et al., 2020).

Cabe destacar que a redução gradual nos parâmetros de função autonômica está relacionada com idade avançada, índice de massa corpórea (IMC) e gordura visceral e foi associada com uma possível disautonomia silenciosa em indlvíduos não diagnosticados com DM, mas com fatores de risco, como demonstrado por Cherkas A, et al. (2015).

Uma metanálise com mais de 2.900 pacientes mostrou que a mortalidade por um período de até dez anos era de $30,4 \%$ em pacientes diabéticos com NAC, detectados pela redução da VFC, e de 13,4\% naqueles sem sinais de NAC pela VFC (MASER RE, et al. 2003). Pacientes com diagnóstico de NAC apresentam risco significativo de intervalos prolongados no intervalo QT, associados a mortes súbitas (WHITSEL EA, et al., 2000).

Com base nos textos científicos, é preciso considerar uma avaliação minuciosa dos testes de diagnóstico como frequência Cardíaca de repouso, variabilidade da frequência cardíaca e teste de exercício de 4 segundos, a fim de se obter uma análise estatística de confiança. (WHITSEL EA, et al., 2000). As características dos pacientes com diabetes mellitus, no atual estudo, estão relacionadas à precisão da análise dos dados, uma vez que a presente pesquisa foi desenvolvida seguindo critérios rigorosos de análise, constitui-se dados fidedignos sobre os pacientes avaliados.

Além disso, é necessário apontar que não houve nenhum tipo de falha na elaboração do manuscrito, fato que propicia maior segurança sobre os dados quantitativos informados pela pesquisa. Dessa forma, a atual pesquisa deve ser considerada com elevada especificidade para os casos de análise do sistema nervoso em indivíduos jovens com diabetes mellitus.

Alterações nos índices de VFC são preditores de adaptação anormal e insuficiente do SNA, fator este que aumenta o risco de morte súbita por arritmias cardíacas e está associada à elevação das taxas de mortalidade por outras causas. A disfunção autonômica cardíaca em pacientes já em risco, como no DM, pode ser um fator complicador neste grupo de pacientes (SCHIMID H, et al., 2007).

Os resultados obtidos no presente estudo, corroboram os achados de Abd El Dayem SM, et al (2011), que investigaram o mesmo fenômeno em indivíduos com DM1, sugerindo indícios precoces de alteração parassimpática. Karamitsos DT, et al. (1998), verificaram em um estudo prospectivo, a história natural da progressão da neuropatia autonômica cardiovascular em um período de dois anos, com medidas repetidas a cada três meses através de testes padrão para diagnóstico desta condição clínica, importante deterioração do ramo parassimpático em relação ao ramo simpático, em seu período de seguimento, contudo sem diferenças significativas nos três primeiros meses. 
Cabe aqui destacar, que o referido estudo não utilizou um instrumento para avaliar isoladamente a AVC, somente o balanço simpatovagal, diferentemente deste estudo, que usou um instrumento validado e fidedigno para avaliação do ramo parassimpático (EWING D, CLARKE B, 1982).

Apesar do presente estudo não encontrar diferenças AVC e na VFC, isso pode ser explicado em grande parte pelo tamanho amostral e pela limitação dos índices do domínio do tempo e da frequência obtidos pela VFC, apesar deste método ser amplamente utilizado pela comunidade científica (ZIEGLER D, et al., 1992 e KIM SY, EULER DE, 1987).

De acordo com Jaiswal M, et al (2013), uma redução significativa dos índices SDNN, rMSSD, com redução importante da VFC, foi observada por quando compararam jovens com DM1 com grupo controle de indivíduos sem diabetes, assim como demonstrando ser significativa a progressão da neuropatia autonômica cardiovascular durante os dois anos posteriores ao seu diagnóstico.

Dessa forma, estudos de maior follow-up em relação a temática alertam sobre a importância do diagnóstico precoce das alterações do SNA em especial a AVC em indivíduos com DM1, demonstrando piora do mecanismo de lesão neurológica principalmente em função do tempo de exposição à doença, contudo nenhum destes avaliou de forma isolada o ramo parassimpático (MAY O, ARILDSEN H, 2011 e ZANONE MM, et al., 2003).

É importante ressaltar, que o ramo parassimpático tende a ser o primeiro ramo do SNA a ser acometido na progressão natural da doença, sendo por este motivo válida a utilização do T4s em nosso estudo, já que - IVC é um índice que reflete a magnitude da retirada vagal cardíaca frente a uma condição de estresse fisiológico e que avalia isoladamente o ramo parassimpático do SNA cardíaco, podendo inclusive ser utilizado como um preditor importante neste tipo de investigação, já que é facilmente reprodutível, de baixo custo e de simples execução (ARAÚJO CGSD, et al., 2003 e PAIVA VCD, et al., 2011). Neste sentido, destaca-se a importância da investigação da AVC nos estágios iniciais após diagnóstico.

Considerando que a presença de alterações na modulação do SNA estejam associadas com um aumento do risco para eventos cardiovasculares, e que o ramo parassimpático tende a ser o primeiro acometido no início da doença, o rastreamento de alterações do SNA por meio de testes para avaliação da nos estágios iniciais do diabetes podem contribuir para evitar maiores comprometimentos e agravos, principalmente no que tange a AVC (VINIK Al, ZIEGLER D, 2007; TESFAYE S, et al., 2010 e VINIK Al, et al., 2013).

Cabe destacar, que embora exista na literatura testes específicos para investigação de disfunção autonômica em indivíduos com DM Ewing D e Clark B (1982), no presente estudo optamos por realizar o T4s e a VFC, uma vez que o propósito não era de diagnosticar NAC. Além disso, os dois testes que foram utilizados, também são válidos e amplamente utilizados e descritos na literatura científica, tendo ainda uma boa aplicação prática (ARAÚJO CGSD, et al., 2003 e PAIVA VCD, et al., 2011). Diante do exposto, destacase no presente estudo como ponto forte, a avaliação da AVC isoladamente pelo t4s expresso pelo IVC, um teste simples validado farmacologicamente, fidedigno e de fácil aplicação (PAIVA VCD, et al., 2011).

Tendo em vista que o diagnóstico precoce de disfunção autonômica em pacientes diabéticos pode reduzir de forma importante os desfechos de mortalidade por causas cardiovasculares, uma vez que a NAC constitui um importante marcador de risco para macroangiopatia em geral e para doença arterial coronariana, a proposta deste teste como investigação e rastreio de disfunção autonômica na prática diária pode ser útil, principalmente pela sua validade externa.

\section{CONCLUSÃO}

Foram observadas diferenças na FC de repouso em indivíduos com DM1 quando comparados ao controle. Entretanto, em relação a VFC e AVC não foram encontradas diferenças significativas, apesar da AVC estar menor no grupo GDM1. Por fim, destaca-se que o T4s é um teste de fácil execução e boa validade externa que pode ser incluído como um método de rastreio de disfunção autonômica em pacientes com DM, em especial por investigar de forma isolada o ramo parassimpático, que parece ser o primeiro a apresentar comprometimento pela neuropatia diabética. Dessa forma, os achados do presente estudo, contribuem por reforçar que identificar de forma precoce a disfunção autonômica no DM, pode acelerar a profilaxia e o controle de lesões em órgãos alvos com o uso de drogas específicas e também com abordagens não farmacológicas. 


\section{REFERÊNCIAS}

1. ABD EL DAYEM SM, et al. Natural progression of cardiac autonomic neuropathy in patients with type 1 diabetes: a four-year follow-up study. Anadolu Kardiyol Derg. 2011; 11(3): 224-31.

2. ARAÚJO CGSD, et al. Intra and interdays reliability of the 4-second exercise test. Rev Bras Med Esporte. 2003; 9(5):293-298.

3. BREDER ISS, Sposito AC. Cardiovascular autonomic neuropathy in type 2 diabetic patients. Rev. Assoc. Med. Bras. vol.65 no.1 São Paulo Jan. 2019.

4. CHERKAS A, et al. The correlations of glycated hemoglobin and carbohydrate metabolism parameters with heart rate variability in apparently healthy sedentary young male subjects. Redox Biol. 2015;5:301-7.

5. DE ANGELIS K, et al. Diabetes and cardiovascular autonomic dysfunction: application of animal models. Auton Neurosci. 2009; 145(1-2):3-10.

6. EWING D, CLARKE B. Diagnosis and management of diabetic autonomic neuropathy. Br Med J (Clin ResEd. 1982; 285(6346): 916.

7. GARBER CE, et al. Quantity and quality of exercise for developing and maintaining cardiorespiratory, musculoskeletal, and neuromotor fitness in apparently healthy adults: guidance for prescribing exercise. Med Sci Sports Exerc. 2011; 43(7):1334-59.

8. HOWORKA K, et al. Effects of physical training on heart rate variability in diabetic patients with various degrees of cardiovascular autonomic neuropathy. Cardiovasc Res. 1997;34(1):206-14.

9. JAISWAL M, et al. Reduced heart rate variability among youth with type 1 diabetes: the SEARCH CVD study. Diabetes Care. 2013; 36(1):157-62.

10. JAYORKA M, et al. Parasympathetic versus sympathetic control of the cardiovascular system in young patients with type 1 diabetes mellitus. Clin Physiol Funct Imaging. 2005; 25(5):270-4.

11. KARAMITSOS DT, et al. The natural history of recently diagnosed autonomic neuropathy over a period of 2 years. Diabetes Res Clin Pract. 1998; 42(1):55-63.

12. KIM SY, EULER DE. Baroreflex sensitivity assessed by complex demodulation of cardiovascular variability. Hypertension. 1997; 29(5):1119-25.

13. MACHADO CA, et al. V Diretrizes Brasileiras de Hipertensão. Arq Bras Cardiol. 2007; 89(3): e24-e79

14. MASER RE, et al. The association between cardiovascular autonomic neuropathy and mortality in individuals with diabetes: a meta-analysis. Diabetes Care. 2003; 26(6):1895-901.

15. MAY O, ARILDSEN H. Long-term predictive power of simple function tests for cardiovascular autonomic neuropathy in diabetes: a population-based study. Acta Diabetol. 2011; 48(4):311-6.

16. PAIVA VCD, et al. Comparison of assessment methods of cardiac vagal modulation. Arq Bras Cardiol. 2011; 97(6):493-501.

17. POP-BUSUI R, et al. Effects of cardiac autonomic dysfunction on mortality risk in the Action to Control Cardiovascular Risk in Diabetes (ACCORD) trial. Diabetes Care. 2010; 33(7):1578-84.

18. ROLIM LC, et al. Diabetic cardiovascular autonomic neuropathy: risk factors, clinical impact and early diagnosis. Arq Bras Cardiol. 2008; 90(4): e24-31.

19. SCHIMID H. [Cardiovascular impact of the autonomic neuropathy of diabetes mellitus]. Arq Bras Endocrinol Metabol. 2007; 51(2):232-43.

20. SPALLONE V, et al. Cardiovascular autonomic neuropathy in diabetes: clinical impact, assessment, diagnosis, and management. Diabetes Metab Res Rev. 2011; 27(7):639-53.

21. TESFAYE S, et al. Diabetic neuropathies: update on definitions, diagnostic criteria, estimation of severity, and treatments. Diabetes Care. 2010; 33(10):2285-93.

22. VANDERLEI LC, et al. Basic notions of heart rate variability and its clinical applicability. Rev Bras Cir Cardiovasc. 2009; 24(2):205-17.

23. VINIK AI, ZIEGLER D. Diabetic cardiovascular autonomic neuropathy. Circulation. 2007; 115(3):387-97.

24. VINIK AI, et al. Diabetic cardiac autonomic neuropathy, inflammation and cardiovascular disease. J Diabetes Investig. 2013; 4(1):4-18.

25. ZANONE MM, et al. Autonomic function and autoantibodies to autonomic nervous tissues at follow-up in a cohort of young patients with type 1 diabetes. Effects of serum from diabetic patients on human adrenergic cells. $J$ Neuroimmunol. 2003; 139(1-2):66-75.

26. ZIEGLER D, et al. Assessment of cardiovascular autonomic function: age-related normal ranges and reproducibility of spectral analysis, vector analysis, and standard tests of heart rate variation and blood pressure responses. Diabet Med. 1992; 9(2):166-75.

27. WHITSEL EA, et al. Reassessing the role of QT(c) in the diagnosis of autonomic failure among patients with diabetes: a meta-analysis. Diabetes Care. 2000;23(2):241-7. 